\title{
An Analysis of the Evidence Base Relating to the Role of Warm-Up and Stretching in Reduction of Injury Risk in Athletes
}

\author{
Manan Vora* and Manit Arora \\ Fortis Hospital, Mohali, India \\ *Corresponding author: Manan Vora, Fortis Hospital, Mohali, India
}

\begin{abstract}
Pre-participation warm up and stretching - in various forms - is widely employed by athletes before both training and competition. Various potential benefits of warming up and stretching are proposed, including a reduction in the risk of injury. Our aim is to summarize the available literature, identify appropriate information resources, and to produce a reasoned evaluation of the available evidence. We concluded that every athlete, coach, or conditioning trainer must incorporate a warm-up protocol along with a stretching routine in an athlete's training regimen.
\end{abstract}

\section{Introduction}

Athletes, coaches, trainers, physiotherapists, and physicians recommend warm-up, stretching and cool down exercises in an effort to both prevent injury and enhance performance [1]. Warmup increases blood flow to muscles, speed of nerve impulses, oxygen and energy substrate delivery, and oxygen release from hemoglobin and myoglobin [2]. It decreases both the activation energy for cellular reactions and muscle viscosity [2]. Warm-up is designed to increased muscle/tendon suppleness, increase body temperature, and enhance free, coordinated movement [3]. Warm-up techniques are classified in 3 major categories: (a) passive warm-up-increases temperature by some external means; (b) general warm-up increases temperature by nonspecific body movements; and (c) specific warm-up - increases temperature using similar body parts that will be used in the subsequent, more strenuous activity $[4,5]$. Over the years, warm up protocols consisting of the abovementioned categories along with various structures (e.g. varied intensity, duration and recovery) have been used [6].

Muscular injury is one of the major problems facing today's athletes, both recreational and professional, with injuries to skeletal muscle representing more than $30 \%$ of the injuries seen in sports medicine clinics [7]. There has been research done in the past both for and against the need for warm-up and stretching before sporting activity, and its role in injury prevention.

\section{Evidence for Warm-Up}

Studies have shown that the benefits of warm-up potentially reduce the risks of strain injury to the muscle [8]. Several programs that combine warm-up, strength training, balance training, stretching, controlled rehabilitation, information about the importance of disciplined play and the increased risk of injury, and correction and supervision by doctor(s) and physiotherapist(s) have demonstrated effectiveness in the prevention of knee and ankle injuries [9-11]. It has also been established that during preseason screening and rehabilitation following hamstring muscle injury, clinicians should consider the influence of hamstring strength, flexibility, warm-up, and fatigue on muscle performance [12]. A study using biomechanical support to assess the athletic practice of warming up prior to an exercise task to reduce the incidence of injury, inferred that physiologic warming is of benefit in preventing muscular injury by increasing the length and elasticity of the muscle-tendon unit [13]. 


\section{Evidence Against Warm-Up}

On the flip side, certain authors have said there is no evidence of muscle strain or injury resulting from performance without warm-up [14]. One study concluded that warm-up does not prevent Delayed Onset Muscle Soreness resulting from exhausting exercise [15]. Certain authors suggested that passive warm-up performed before eccentric exercise may be more beneficial than active warmup or no warm-up in attenuating swelling, but do not support the use of warm-up to prevent, attenuate, or resolve more quickly the strength loss, loss of motion, or soreness resulting from eccentric muscle damage [16].

\section{Warm Up and Stretching}

Preparation for athletic activity often includes both stretching and warm-up, making it difficult to assess their independent effects on injury prevention [17]. Research conveys that certain techniques and protocols have shown a positive outcome on deterring injuries. Warm-up and stretching protocol should be implemented prior to physical activity, with the routine allowing stretching to occur within 15 minutes immediately prior to the activity, in order to receive the most benefit [7]. Stretch reduces passive tension, and benefits from lower tension are reduced sensations of stiffness and soreness, thus presenting a new proposal for passive stretches as a warm-up strategy [18]. Studies showed that only ankle dorsiflexion was influenced by warming up [19], whereas hip and knee flexibility increased with stretching [20]. Warm-up and stretching are essential to preventing muscle injuries by increasing the elasticity of muscles and smoothing muscular contractions, however improper or excessive stretching and warming up can predispose to muscle injury [2]. In contrast to other studies, some authors found the combination of warm-up, stretching and massage to reduce some negative effects of eccentric exercise, but the results were inconsistent [21]. However, one author dismissed stretching saying that because most injuries occur during eccentric contractions within the normal range of joint motion, it is not clear how increasing the range of motion through stretching will decrease injury risk [22].

\section{Summary of Evidence}

There is not sufficient evidence to strictly endorse or discontinue warm-up before sporting activity, however there is adequate evidence suggesting its role in prevention of certain muscle injuries. Even the studies that disregard the role of warm-up, do not mention any significant demerit or increased risk of injury if an athlete were to undergo a supervised and correct warm-up protocol, and hence every athlete, coach, or conditioning trainer must incorporate it in an athlete's training routine. An ideal warm-up session should include raising body parameters, activating muscles, mobilising joints, as well as preparing an athlete for the activity they perform in their particular sport. Incorporating stretching along with warmup could be beneficial. In setting up a safe stretching program, one should (A) precede stretching exercises with a mild warm-up; (B) use static stretching; (C) stretch before and after a workout; (D) begin with mild and proceed to moderate exercises; (E) alternate exercises for muscle groups; (F) stretch gently and slowly until tightness, not pain, is felt; and (G) hold the position for 30 to 60 seconds [23]. A correct attitude and mind set towards warm-up is also essential. A study showed subjects with a more favourable attitude toward warm-ups performed significantly better, while those subjects with a less favourable attitude did not improve significantly when warm-ups were given [24].

\section{Conclusion}

Injury prevention (and reducing the risk of injury) is one of the most important aspects in sports medicine today. Therefore, wider promotion of injury prevention resources, combined with research into the effectiveness of these resources and how players can be encouraged to adopt appropriate injury prevention strategies, is recommended [25]. Better research is needed to determine the role of warm-up and stretching in sport, and its impact on injury prevention.

\section{References}

1. (1998) American College of Sports Medicine Position Stand. The recommended quantity and quality of exercise for developing and maintaining cardiorespiratory and muscular fitness, and flexibility in healthy adults. Med Sci Sports Exerc 30(6): 975-991.

2. Safran MR, Seaber AV, Garrett WE (1989) Warm-Up and Muscular Injury Prevention an Update. Sports Med 8(4): 239-249.

3. Smith CA (1994) The Warm-Up Procedure: To Stretch or Not to Stretch. A Brief Review. J Orthop Sports Phys Ther 19(1): 12-17.

4. Shellock FG (1983) Physiological Benefits of Warm-Up. Phys Sportsmed 11(10): 134-139.

5. Shellock FG, Prentice WE (1985) Warming-Up and Stretching for Improved Physical Performance and Prevention of Sports-Related Injuries. Sports Med 2(4): 267-278.

6. Bishop D (2003) Warm Up II. Sports Med 33(7): 483-498.

7. Woods K, Bishop P, Jones E (2007) Warm-up and stretching in the prevention of muscular injury. Sports Med Auckl NZ 37(12): 1089-1099.

8. Garrett WE (1996) Muscle strain injuries. Am J Sports Med 24(6 Suppl): S2-8.

9. Ekstrand J, Gillquist J, Liljedahl SO (1983) Prevention of soccer injuries. Supervision by doctor and physiotherapist. Am J Sports Med 11(3): 116120.

10. Hewett TE, Lindenfeld TN, Riccobene JV, Noyes FR (1999) The effect of neuromuscular training on the incidence of knee injury in female athletes. A prospective study. Am J Sports Med 27(6): 699-706.

11. Wedderkopp N, Kaltoft M, Lundgaard B, Rosendahl M, Froberg K (1999) Prevention of injuries in young female players in European team handball. A prospective intervention study. Scand J Med Sci Sports 9(1): 41-47.

12. Worrell TW, Perrin DH (1992) Hamstring muscle injury: the influence of strength, flexibility, warm-up, and fatigue. J Orthop Sports Phys Ther 16(1): 12-18.

13. Safran MR, Garrett WE, Seaber AV, Glisson RR, Ribbeck BM (1988) The role of warmup in muscular injury prevention. Am J Sports Med 16(2): 123-129. 
14. Massey BH, Johnson WR, Kramer GF (1961) Effect of Warm-Up Exercise upon Muscular Performance Using Hypnosis to Control the Psychological Variable. Res Q Am Assoc Health Phys Educ Recreat 32(1): 63-71.

15. High DM, Howley ET, Franks BD (1989) The effects of static stretching and warm-up on prevention of delayed-onset muscle soreness. Res Q Exerc Sport 60(4): 357-361.

16. Evans RK, Knight KL, Draper DO, Parcell AC (2002) Effects of warm-up before eccentric exercise on indirect markers of muscle damage. Med Sci Sports Exerc 34(12): 1892-1899.

17. Thacker SB, Gilchrist J, Stroup DF, Kimsey CD (2004) Clinical Sciences Clinical Investigations The Impact of Stretching on Sports Injury Risk: A Systematic Review of the Literature. Med Sci Sports Exerc 36(3): 371378.

18. Reisman S, Walsh LD, Proske U (2005) Warm-up stretches reduce sensations of stiffness and soreness after eccentric exercise. Med Sci Sports Exerc 37(6): 929-936.

19. Wiktorsson-Möller M, Oberg B, Ekstrand J, Gillquist J (1983) Effects of warming up, massage and stretching on range of motion and muscle strength in the lower extremity. Am J Sports Med 11(4): 249-252.
20. Hubley CL, Kozey JW, Stanish WD (1984) The effects of static stretching exercises and stationary cycling on range of motion at the hip joint. J Orthop Sports Phys Ther 6(2): 104-109.

21. Rodenburg JB, Steenbeek D, Schiereck P, Bär PR (1994) Warm-up, stretching and massage diminish harmful effects of eccentric exercise. Int J Sports Med15(7): 414-419.

22. Shrier I (1999) Stretching before exercise does not reduce the risk of local muscle injury: A critical review of the clinical and basic science literature. Clin J Sport Med Off J Can Acad Sport Med 9(4): 221-227.

23. Beaulieu JE (1981) Developing a Stretching Program. Phys Sportsmed 9(11): 59-69.

24. Smith JL, Bozymowski MF (1965) Effect of Attitude toward Warm-Ups on Motor Performance. Res Q Am Assoc Health Phys Educ Recreat 36(1): 78-85.

25. Hume PA, Steele JR (2000) A preliminary investigation of injury prevention strategies in Netball: are players heeding the advice? J Sci Med Sport 3(4): 406-413.
This work is licensed under Creative Commons Attribution 4.0 License

To Submit Your Article Click Here:

Submit Article

DOI: $10.32474 /$ OSMOAJ.2019.02.000144

$\begin{gathered}\text { Orthopedics and Sports Medicine } \\ \text { Open Access Journal }\end{gathered}$
Assets of Publishing with us
- Global archiving of articles
- Immediate, unrestricted online access
- Rigorous Peer Review Process
- Authors Retain Copyrights
- Unique DOI for all articles

